\title{
Hydrodynamic Analysis and Irrigation Device Design for the Coaxial and Bimanual Phacoemulsification Techniques in Cataract Surgery
}

\author{
Yury Spirochkin \\ Engineering Centre of Nuclear Equipment Strength (ENES) \\ Russia
}

\section{Introduction}

The phacoemulsification method, being in use in cataract surgery, includes mechanical disintegration of the lens of the human eye by special tools introduced into the anterior chamber of the eye through small incisions. The generated fragments of the lens are removed together with the intraocular fluid by aspiration. An artificial lens of transparent plastic is then inserted into the eye to replace the eliminated lens.

To avoid collapse of the anterior chamber because of the rarefaction (collapse may cause irreversible tissue injury), irrigation is carried out simultaneously with aspiration using a replacing fluid. Several phacoemulsification techniques are elaborated. Key ways in which they differ one from another are in the method of lens disruption and in the organization of aspiration-irrigation flow. Each technique requires adequate surgical tips to the instrument. The basic technique now widely used is a combination of eye lens disintegration, aspiration and irrigation functions in one tip made of two coaxial tubules. The inner tubule is periodically subjected to longitudinal oscillations with an ultrasonic frequency in order to crush the lens fragments situated near the tubule end. The emulsion formed by these fragments and the fluid filling the anterior chamber are eliminated trough this tubule. The irrigation fluid is brought into the chamber through the annular channel between the inner and external tubules (Fig. 1).

Bimanual phacoemulsification is an alternative technique based on the use of two separated tips: one for aspiration and the other for irrigation. They are introduced into the anterior chamber through two incisions separated by a distinct distance and positioned angularly (Fig. 2). As a result, the fluid flow pattern across the surgical field changes substantially in comparison with the basic (coaxial) technique.

The decision to perform coaxial phacoemulsification is by many ophthalmic surgeons influenced by the greater experience with this technique and the desire of surgeons to avoid additional incisions. It was however observed in a series of operations that the bimanual technique is accompanied by a decrease of adverse hydrodynamic effect on eye tissues and of irrigation fluid consumption (Trubilin et al., 2005). A reduction of operation time was also observed. It was supposed that these favorable tendencies (which directly effect the quality 
of ophthalmic surgical care) are caused by the differences mentioned above in the intraocular hydrodynamics.

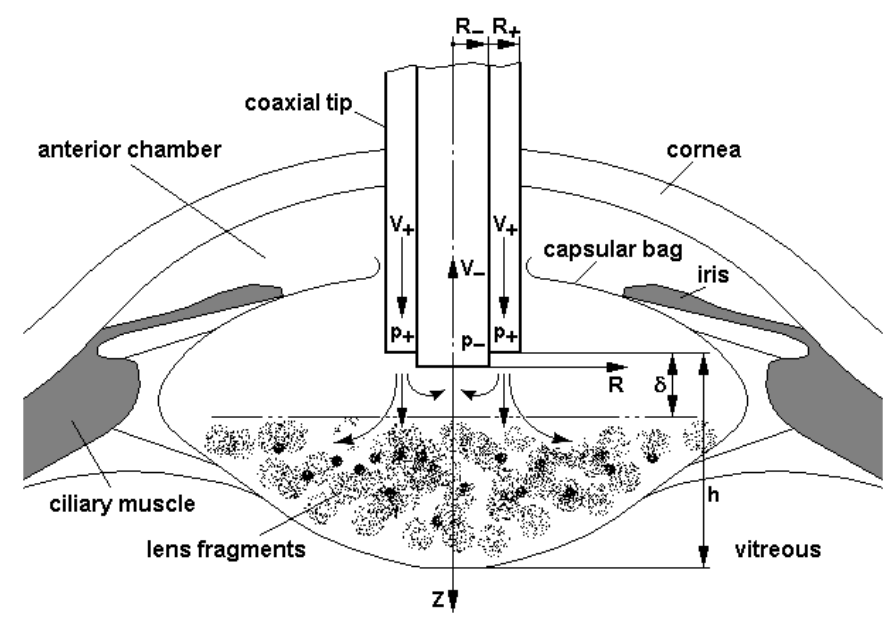

Fig. 1. Flow in the coaxial phacoemulsification technique

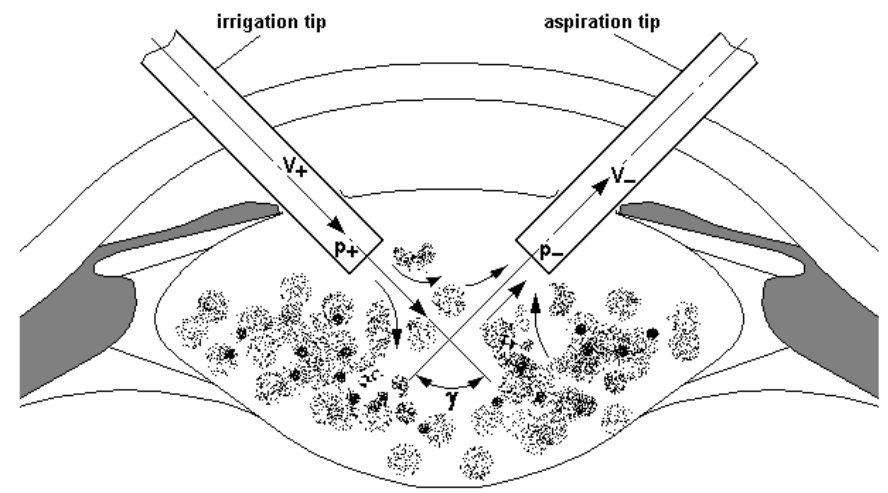

Fig. 2. Flow in the bimanual phacoemulsification technique

The possibilities of experimental validation of this assumption on natural eye specimens are limited because of high preparation costs, difficulties in physical data recording within small volumes, etc. Under these circumstances, mathematical modeling is the most accessible method for study of intraocular fluid flow.

Generally, both the empirical approach to the choice of ophthalmic surgical tools, which is widespread now, and the subjectivism in the clinical appraisal of their advantages or disadvantages lead to difficulties in design and development of tools which could ensure minimal tissue damage and maximal efficiency of the corresponding operation technique. Therefore, analysis of a new surgical method or a technique and the tools that will be used therein by means of an adequate mathematical model is quite an urgent problem. Its solution is demanded by ophthalmic surgeons as well as by designers of surgical tools. 
In order to prove the advantages of the bimanual phacoemulsification technique the following steps are necessary:

- building an adequate mathematical model in the form of equations describing the fluid movements in the capsule bag volume during the operation

- clarifying on the basis of solution of these equations the main capabilities of this technique concerning the decrease of the injury from surgery, irrigation fluid consumption and operation time (relative to the coaxial technique)

- determining a set of quantitative parameters that characterize the operation technique and values of these parameters which could ensure its maximal efficiency.

The following parameters are taken as the efficiency criteria: reduction of tissue trauma caused by hydrodynamic forces, lowering of irrigation fluid consumption and decrease of the operation time. The tissue trauma can be associated with, amongst other causes:

- $\quad$ high fluid pressure including the dynamic sinusoidal and pulsation components

- inadvertent entry of the posterior lens capsule into the aspiration tubule

- collapse of the capsule chamber resulting from high difference between the internal and external pressure.

Hense, the required mathematical model should allow determination of the hydrodynamic pressure in zones of the anterior eye (as a trauma index) and the irrigation fluid consumption (as an economic index) as well as evaluation of the operation time.

In this Chapter, a simplified analytical model and analysis of hydrodynamic processes during phacoemulsification are presented; on the basis of the analysis, advantages of the bimanual technique are revealed and the favourable effect of this technique on operation time is evaluated. Subsequently, a review of applications of the sophisticated numerical analysis method - the finite element method (FEM) - applied to ocular biomechanics is given; use of this method for simulation of cataract surgery techniques could make analytical results more exact. As a practical embodiment of the ideas of the hydrodynamic analysis, designs of irrigation devices intended for the bimanual phacoemulsification technique are considered.

\section{Simplified mathematical model of hydrodynamic processes}

Mathematical modeling of the intraocular fluid flow is based on the mass, impulse and energy conservation laws. Assuming some simplifications, these laws allow series of relations to be obtained between the required values of pressure and irrigation fluid consumption, and the known geometric, physical and mechanical parameters. The fluid is assumed as incompressible viscose medium under stationary flow conditions. This assumption is valid even when oscillations of irrigation fluid rate exist over a wide frequency range. Indeed, the frequency value $f$, over which the non-stationary wavepropagation processes must be taken into account, can be evaluated by the formula

$$
f \approx \frac{c}{L}
$$

where $c$ is the acoustic speed in the irrigation fluid, and $L$ denotes the characteristic dimension of the flow domain.

As to the eye, $L$ is about $10 \mathrm{~mm}$, while $c$ is about $1000 \mathrm{~m} / \mathrm{s}$. Therefore, the threshold value of $f$ is not less than $10^{5} \mathrm{~Hz}$, and the slow dynamic processes in consideration can be regarded as stationary ones. 
When building an adequate mathematical model, it is important to determine whether the fluid flow is turbulent or laminar one. The boundary between both flow types is determined by the Reynolds criterion

$$
\operatorname{Re}=\frac{\rho L V}{v}
$$

where $\rho$ is the density of the fluid; for the irrigation fluid $\rho=1000 \mathrm{~kg} / \mathrm{m}^{3}$, for the emulsion $\rho$ $=998 \mathrm{~kg} / \mathrm{m}^{3}$ (Malygin, 2002); $V$ is the flow velocity of the irrigation fluid and of the emulsion; it ranges from 0.2 to $0.6 \mathrm{~m} / \mathrm{s}$; the mean value is $0.4 \mathrm{~m} / \mathrm{s}$ (Malygin, 2002); $v$ denotes the dynamic viscosity coefficient; for the aqueous $v=0.014 \mathrm{~Pa} s$ (Malygin, 2002).

In the case of the irrigation fluid outflow, the characteristic dimension $L$ is the tubule diameter, up to $3 \mathrm{~mm}$. Based on these values, $R e$ is not more than 90 , this is far from the critical value $\operatorname{Re}_{C}=2300$ typical for tube flows. Therefore, the irrigation fluid flow is laminar one. For the emulsion flow, $L$ can be taken equal to capsular bag diameter $(10 \mathrm{~mm})$, so the Reynolds criterion can reach 300 . However even in this case, it is substantially lower than the critical value quoted just now, or typical for free flow $\left(\operatorname{Re}_{\mathrm{C}}=10^{5}\right)$. The introduction of viscoelastic substances essentially increases the emulsion viscosity (Malygin, 2002), and this leads to corresponding decrease of the Reynolds criterion. Similar evaluation is also valid for the aspiration flow. Consequently, there is no reason to speak about the presence of flow instability, i.e. the appearance of turbulence in the irrigation and aspiration flows, and also in the emulsion within the operation field.

The laminar character of the flows under consideration makes their mathematical modeling easier. Usually, the equations in partial derivatives, e.g. the Navier-Stocks equations, are used for modeling in hydrodynamics (Landau and Livshits, 1988). They are solved by numerical methods taking into account given boundary conditions. As a result, the required parameters of the fluid flow in different points of the investigated domain are found: pressure, velocity, etc. However, solution of these equations for domains with intricate shapes, such as the anterior chamber of the eye is a difficult problem, especially when taking into account the configurations of the irrigation and aspiration flows, the biphasic character of the emulsion, the spatial diffusion of the lens fragments, the presence of an acoustic (ultrasonic) action, the movements of the surgical instruments, the flexibility of the eye tissues, etc.

Moreover, many physical parameters of the eye taken as input data for mathematical modeling have a significant scattering from one individual to another, and can not be exactly measured in each case with certainty. Additional uncertainties are caused by elasticity (flexibility) parameters of the ocular tissue or position of the aspiration-irrigation tip, which is manipulated by the surgeon's hand. Therefore the model needs to be simplified to obtain approximate solutions with acceptable precision and reasonable computing expenses for the calculations. But every simplification must be carefully grounded - in particular by means of special experiments. Otherwise the calculation errors may become unacceptable high.

Taking these factors into account it may not seem worthwhile creating complicated numerical approximations of the fluid movements. On the contrary, it is reasonable to use simple and evident physical relations resulting from the fundamental conservation laws. A basis for this approach lies correct choice of the surface on which flow parameters are checked, i.e. the checking surface (Abramovich, 1969). If a quantitative correlation between pressure and velocity 
(correspondingly, the flow rate) can be established for various zones of this surface, without going into details concerning the flow in the internal domains, this correlation gives the required solution for each of the analyzed cases. Correspondingly, a comparison of the cases in dependence on the mentioned parameters is possible.

The simplified model described below is intended for comparative analysis of the two techniques of the phacoemulsification method under consideration. It takes into account the most substantial factors in which their differences may arise. In this case, the factors simultaneously inherent to both methods present no interest and are not subjected to the analysis. In particular, this model does not simulate the lens disintegration process, movement/shifting of surgical tools during the operation, finite dimensions of the lens particles, their motions, emulsion inhomogeneity, etc. Therefore it may be called the $1^{\text {st }}$ approach model.

Let us first turn to the more established and in some ways more basic operation technique, i.e. the coaxial technique. The checking surface is chosen as the outlet cross section of the irrigation and aspiration tubules of the tip, which are considered being situated on one plane. The irrigation fluid is introduced into the anterior eye through the external annular part of this surface with the cross section area $S_{+}$with the velocity $V_{+}$. The aspiration of the emulsion is carried out through the internal part with the cross section area $S_{\text {- with }}$ the velocity $V_{-}$. According to the law of continuity, the irrigation volume flow must be equal to the aspirating one, i.e.

$$
S_{+} V_{+}=S_{-} V_{-}
$$

from which is obtained an expression for the aspiration velocity:

$$
V_{-}=\alpha V_{+}, \alpha=\frac{S_{+}}{S_{-}}=\left(\frac{R_{+}}{R_{-}}\right)^{2}-1
$$

where $R_{+}$and $R_{-}$are the internal radii of the irrigation and aspiration tubules, correspondingly.

As follows from the Bernulli equation (which presents one of the forms of the law of conservation of energy), the pressure at the entry into the aspiration tubule comes to

$$
p_{-}=p_{+}-\frac{\rho V_{+}^{2}}{2}\left(\alpha^{2}-1\right)
$$

while at the internal surface of the lens posterior capsule, against the edge of the irrigationaspiration tip, where the irrigation flow completely loses the velocity (on the wall), the pressure value is

$$
p_{c}=p_{+}+\frac{\rho V_{+}^{2}}{2}
$$

The parameter $p_{+}$denotes the static pressure at the irrigation tubule outlet. For the coaxial tip of the above described design, $R_{+}$is about $2-3$ times greater than $R_{-}$. It follows that $a$ may range from 3 up to 8 . The pressure at the entry into the aspiration tubule $p_{-}$, as it seen from formula (4), is significantly lower than the pressure $p_{+}$(about a half if $a=8$ ), while the 
pressure $p_{\mathrm{c}}$ exceeds the latter by the velocity head $\rho V_{+}{ }^{2} / 2$. The approximate pattern for pressure distribution within the fluid volume between the edge of the coaxial irrigationaspiration tip and the posterior lens capsule (the latter is an additional part of the checking surface) is shown in Fig. 3.

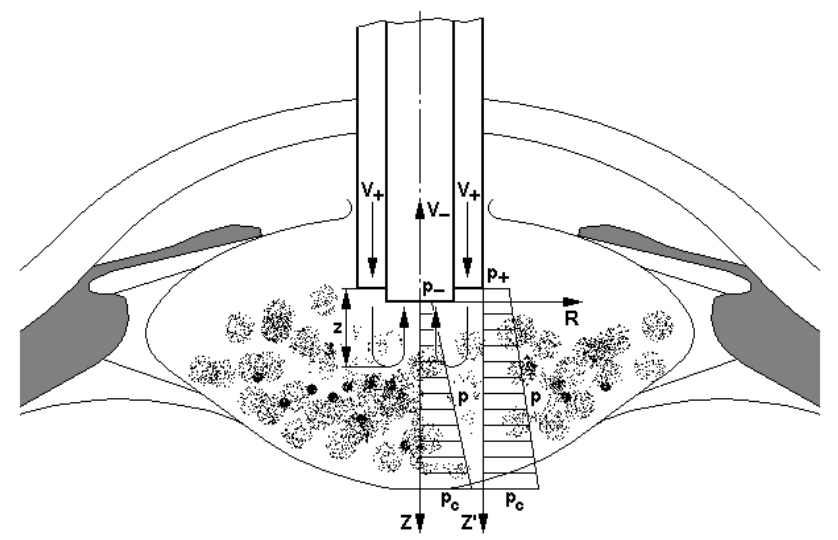

Fig. 3. Pressure distribution (p) and displacement of fluid particles (z) in the coaxial technique

The irrigation flow passes across this volume filled with the lens fragments, mixes with them (forming an emulsion), changes its movement direction into the opposite one and then is sucked into the aspiration tubule. The impulse value change is proportional to the vector difference between the aspiration and irrigation flow velocities and has in this case a maximal value. It produces a load on the internal surface of the capsular bag and can be estimated by the value $p_{\mathrm{c}}$. With the actual pressure distribution a part of the irrigation fluid, moving near the aspiration tubule, has no time to mix with the lens fragments and does not dilute the emulsion, but is at once moved away by the aspiration flow. It does not have any useful effect and is merely pure losses. These shall now be estimated.

The displacement of the irrigation fluid particles along the $Z$ axis (see Fig. 3) up to the stopping, i.e. up to the change of velocity sign, depends on their initial velocity $V_{+}$and on the pressure gradient $\partial p / \partial z$

$$
z=\frac{\rho V_{+}^{2}}{2} \frac{1}{\partial p / \partial z}
$$

On the basis of the obtained pressure distribution (Fig. 3) the $\partial p / \partial z$ value at various points of the area in view can be evaluated, and so the $z$ value can be found. In particular, at $R=R_{-}$

$$
\frac{\partial p}{\partial z} \approx \frac{p_{c}-p_{-}}{h}=\frac{\rho V_{+}^{2}}{2 h} \alpha^{2}
$$

where from follows $z=\frac{h}{\alpha^{2}}$,

and if $R=R_{+}$ 


$$
\frac{\partial p}{\partial z} \approx \frac{p_{c}-p_{+}}{h}=\frac{\rho V_{+}^{2}}{2 h}
$$

then $z=h$

Here, $h$ denotes the distance between the tip edge and the posterior lens capsule (see Fig. 1 ). Thus, the particles of irrigation fluid, which are moving near the internal surface of the irrigation tubule (at $R \approx R_{+}$), pass the entire distance to the posterior lens capsule $(z=h)$. That means they run through the entire emulsion volume, dilute it and are sucked together with the lens fragments. The particles of irrigation fluid, which run directly near the aspiration tubule (at $R \approx R_{-}$), are sharply decelerated and may pass only a much shorter path $\left(z=h / a^{2}\right)$ due to the great pressure drop near the edge. If the lens fragments are situated at a greater distance from the tip edge than $h / a^{2}$, they do not mix with these particles and exit into the aspiration tubule instead of the emulsion.

Assuming a linear pressure distribution along the radial coordinate, the following approximation for the displacement $\mathrm{z}$ of the particles is obtained, depending on the distance from the tubule axis $R$

$$
z(R)=h\left[\frac{1}{\alpha^{2}}+\left(1-\frac{1}{\alpha^{2}}\right) \frac{R-R_{-}}{R_{+}-R_{-}}\right]
$$

Based on this approximate correlation, shown in Fig. 4, and starting from the distance between the tip edge and the conventional boundary of the lens fragments position $\delta$ (see Fig. 1) it is possible to determine the radial coordinate $R(\delta)$ that corresponds to the dividing of the irrigation flux in two parts $Q_{p}$ and $Q_{n}$

$$
R(\delta)=R_{-}+\frac{\frac{\delta}{h}-\frac{1}{\alpha^{2}}}{1-\frac{1}{\alpha^{2}}}\left(R_{+}-R_{-}\right)
$$

The particles, which meet the condition $R>R(\delta)$, form the flux part

$$
Q_{p}=V_{+} \pi\left[R_{+}^{2}-R(\delta)^{2}\right]
$$

which dilutes the emulsion. Therefore it exerts a useful action. The particles with the coordinates $R \leq R(\delta)$ do not reach the emulsion boundary. They form the flux part

$$
Q_{n}=V_{+} \pi\left[R(\delta)^{2}-R_{-}^{2}\right]
$$

which presents pure losses.

As follows from equations (11) - (13), the less the distance between the edge of the irrigation-aspiration tip and the conventional boundary of where the zone with the lens fragments $\delta$ is, the nearer the radial coordinate $R(\delta)$ to the radius value $R_{-}$is, and the less the losses $Q_{n}$ are. Correspondingly, the further the tip edge from the above mentioned boundary lies, the greater the losses are. In particular, for a tip with the parameters $R_{-} \approx 0.5$ $\mathrm{mm}$ and $R_{+} \approx 1.5 \mathrm{~mm}$, when the edge is situated at $2 \mathrm{~mm}$ from the internal surface of capsular bag and at $1 \mathrm{~mm}$ from the indicated boundary, about $40 \%$ of the infused fluid is 
useless taken away through the aspiration tubule. When $\delta=h / a^{2}$ the losses become almost zero. However this distance is difficult to control and it is impossible to avoid losses completely. The calculation shows that losses of the irrigation fluid are a fundamental disadvantage of the coaxial technique, which lowers its efficiency.

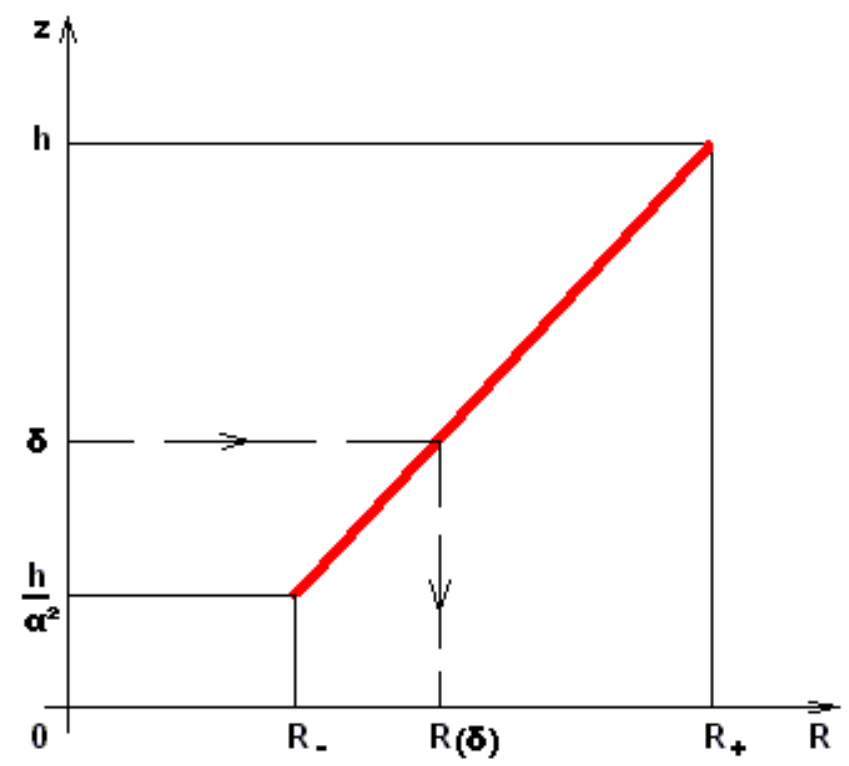

Fig. 4. Displacement of fluid particles, $\mathrm{z}$, in dependence on distance from the tubule axis, $\mathrm{R}$

Let us examine the bimanual technique which uses separated irrigation and aspiration tubules. As done formerly, the control surface will be considered to consist of the tubules' end section areas and of the internal surface of capsular bag (the posterior lens capsule).

If the tubules are positioned parallel (Fig. 5) there is no practical difference in the hydrodynamic situation as compared to the coaxial method.

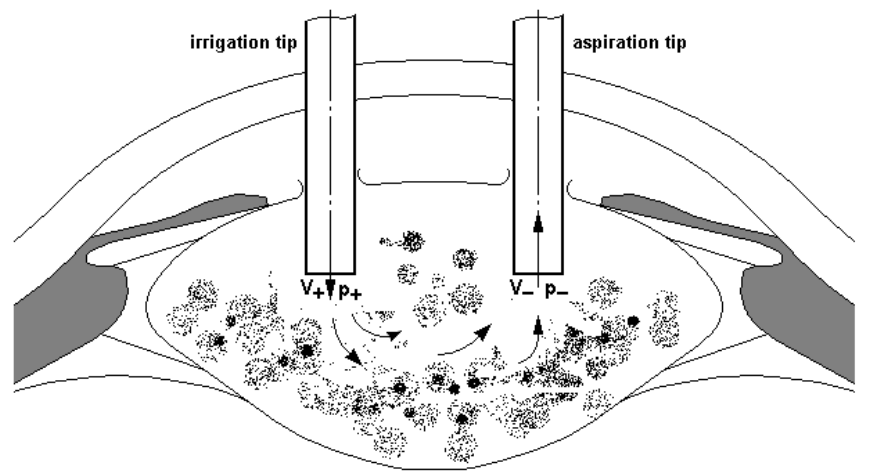

Fig. 5. Bimanual technique: irrigation and aspiration tips are positioned parallel 
The irrigation flow changes the direction into the opposite one, the momentum increment is maximal, and the pressure on the internal surface of capsular bag comes to $p_{\mathrm{c}}=p_{+}+\rho V_{+}{ }^{2} / 2$ as in the precedent example. If the tubules are situated at some angle one to another, the momentum increment decreases proportional to $\cos (\gamma / 2)$, where $\gamma$ denotes the angle between the tubules' axes (see Fig. 2), and the pressure correspondingly decreases. The flatter the tubules are situated one to another, the less is the pressure on the wall. It reaches a minimum value when $\gamma=180^{\circ}$, i.e. the tubules are situated on one axis. Thus the bimanual technique allows control of the pressure by positional relationship of the tubules.

As to the irrigation fluid consumption, the irrigation and aspiration tubules may be placed at different sites of the volume occupied by the lens fragments. In this case it is possible to direct the flux $Q$ across this volume that insures maximal dilution of the emulsion and its aspiration, practically without losses i.e.

$$
Q_{p}=Q=V_{+} \pi R_{+}^{2}, Q_{n}=0
$$

Thus far the quasi-static component of the fluid pressure has been analyzed. Pulsation of the hydrodynamic pressure under the conditions of laminar flow may be caused by oscillations of fluid consumption only. As for the pulsation due to transient processes, these do not exceed several percents of the flowing fluid velocity head (Aviation acoustics, 1986). In reality, the pulsation component does not appreciable change the velocity of the irrigationaspiration flow and the whole modeled situation. Taking this into account would therefore not exert a substantial influence on the results that have been obtained.

The modeling that has been described shows that the bimanual technique is more efficient than the coaxial one in reducing both the traumatic factor (pressure on the eye tissues) and the irrigation fluid consumption.

\section{Evaluation of operation time}

The time necessary to clean the operative field from the lens fragments in many respects depends on the cleaning method and on the degree of homogeneity of the emulsion formed after the irrigation fluid infusion. Several typical cases of the development of this process may be analyzed.

Case 1. The irrigation and aspiration flows are laminar and cover the entire anterior chamber of the eye. In this case, the emulsion is smoothly eliminated from the operative field and the time of full cleaning $t$ can be determined by the formula

$$
t=\frac{\theta}{Q}
$$

where $\theta$ denotes the volume of the anterior chamber.

Case 2. The emulsion is maintained in a homogeneous state during the entire aspiration process. The necessary degree of homogeneity can be achieved by continuous mixing the fluid. In this case, the concentration of the lens fragments decreases with time governed by exponential law with the exponent proportional to $t$.

Case 3. Laminar flow of the irrigation and aspiration fluids occurs only in a part of the operative field while in the remaining domain stagnation zones are observed. In these zones, the fluid circulates in a circle. In this domain the elimination of the emulsion occurs 
only from the above mentioned area, where the irrigation-aspiration flow (laminar flow with running off) is organized.

Of course, none of the cases mentioned is realized in a pure form, but nevertheless they ensure a qualitative comparison between the cleaning time values for the anterior chamber when using the coaxial and the bimanual phacoemulsification techniques. Case 3 gives the best fit for characterization of the coaxial technique. Here, laminar flow with running off occupies a relatively small part of the operative field. According to the estimation above, under these conditions only somewhat more than a half of the infused irrigation fluid volume is effectively used. To clean the stagnation zones the aspiration tip must be shifted. This necessitates the skill of the surgeon and takes an amount of time also.

The bimanual technique is instead characterized rather by Case 1. Laminar flow with running off occupies the major part of the anterior chamber of the eye and correspondingly of the cloud of lens fragments so that shifting of the tip is not necessary.

When using both the coaxial and the bimanual techniques, any shift of the tip (casual or intentional) leads to mixing of the emulsion. The process then tends to follow the characteristics of Case 2 that results in increasing the cleaning time.

Thus, it may be affirmed that in all cases the cleaning time depends to a great extent on the surgeon's skill. However, the bimanual technique does not need a large tip shift and is potentially less traumatic. Besides, the theoretic cleaning time (as follows from the calculations above), which is determined by the loss of the irrigation fluid, is lower by some ten percent.

\section{Review of finite element analyses}

More precise mathematical modeling of hydrodynamic processes during surgical operation is able to increase the efficiency of the bimanual technique both in lowering pressure further and in obtaining a faster cleaning of the operating field from lens fragments. For modeling, the sophisticated numerical approximations based on FEM are applicable.

A short summary review of finite element simulations applied to several problems of eye biomechanics is presented below; it gives information on how investigations develop and which issues arise. The summary does not pretend to be a comprehensive review of the topic and only touches on some key achievements.

One of the first applications of FEM to modeling of the human eye seems to have been carried out by Pinsky and Datye (Pinsky \& Datye, 1991). A finite element model was built to examine effects of radial keratotomy. It assumed horizontal and vertical corneal symmetry and linear elastic material for mechanical behavior of eye tissues.

Bryant and his colleagues have used finite element analysis to investigate the susceptibility of the human cornea with fully healed radial keratotomy incisions (Bryant et al., 1994). The material was assumed to be homogeneous, isotropic, and nonlinearly elastic, with the elastic modulus defined as the derivative of the average stress-strain curve of control strips cut from fresh human donor corneas subjected to tensile testing and failure. Differences between analysis and experimental data have been observed, accounted for by variation in stiffness between the uncut stroma and the wound collagen, and the effect of stress concentration at the wound.

Researchers from the Department of Ophthalmology, Yokohama City University School of Medicine, have applied FEM to determine mechanical behaviour of human eye when loading by impacts of foreign bodies causing intraocular injuries (Uchio at al., 1999). Solid 
modeling was based on information obtained from cadaver eyes. Blunt-shaped missile impacts onto the surface of the cornea or sclera at velocities 30 to $60 \mathrm{~m} / \mathrm{s}$ have been simulated; simulations have been carried out using a supercomputer.

In sequential investigations, finite element modeling technique of the eye has been developed; the main developments were the following: improved approximation of complex geometry (Belezza et al., 2000), (Sigal et al., 2004), anisotropic fiber-matrix material properties (Amini and Barocas, 2009), and nonlinear anisotropic, inhomogeneous, collagen fiber-reinforced structure of eye tissues (Girard et al., 2009). For modeling and calculations, ANSYS software is widely used now. All the investigations noted above focused on improved approximations of eye tissues without adequite modeling of intraocular fluid.

In the Department of Biomechanical Engineering in University of Minnesota Supercomputing Institute, a coupled-flow finite element model of the iris and the aqueous humor has been developed (Elastohydrodynamics of the Anterior Eye, 2002). This model accounts for the passive deformation of the solid iris in response to flow of aqueous humor fluid. The problem is fully coupled because the location of the iris depends on the flow pressure, but the fluid flow pattern depends on the location of the iris (Fig. 6). This relative simple model is already large enough to require considerable computational resources because of the fluid-solid coupling, and the rapidity of ocular events (the lens accommodates from far-viewing to near-viewing in a few tenths of a second).

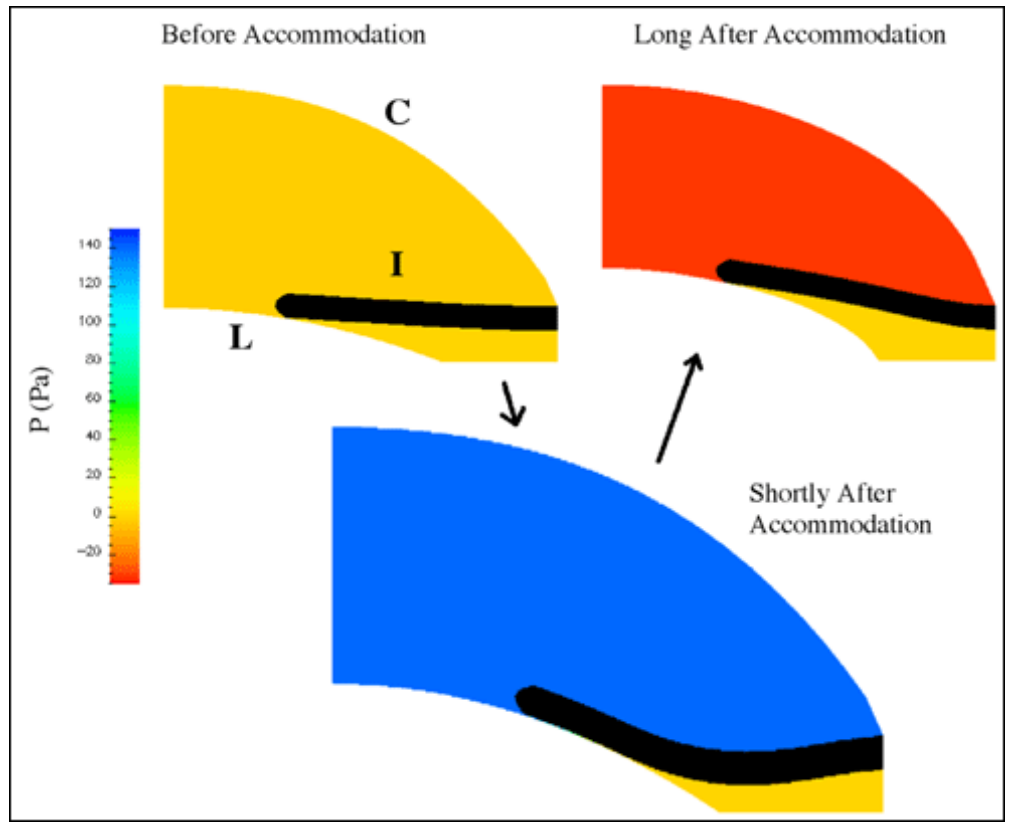

Fig. 6. Iris deformation in response to lens motion (Elastohydrodynamics of the Anterior Eye, 2002)

This coupled-flow finite element model does not cover cataract surgery. An approximation relating to cataract surgery was developed in order to predict changes in the corneal curvature after surgery based on investigations into the material properties of the cornea 
(Crouch et al., 2005). A linear elastic material model was used for the entire eye. To examine the effect of surgical incisions, a cut in the initial eye mesh was made representing the incision made in an extra-capsular cataract extraction procedure. The authors note that extensive future work is needed to produce and validate a biomechanical model that could be used to help predict surgical outcomes. This work may expand on the current work in the following ways:

- $\quad$ apply an oriented fiber tissue model

- augment the finite element model with a time-dependent simulation of the healing response

- $\quad$ experiments with individualized eye models.

With participation of the author of this Chapter, a group of researchers at the Helmholtz Moscow Research Institute of Eye Diseases have started with investigation of accommodation processes using FEM (Poloz et al., 2008).

Scientists and engineers of University of Minnesota now run patient-specific computational fluid dynamics (CFD) and finite element simulations to support medical device innovation and validation (Medical Device Invention and Evaluation through Simulation, Visualization, and Interactive Design, 2010). Simulation could reduce the time it takes to reach bench tests and animal trials, make these trials more effective, and, in time, overtake these methods as the primary evaluative approach in device design. A group of researchers have begun to work on design environment composed of coupled components for running simulations, visualization and comparing massive numbers of results, and interacting directly with the parameter space to explore new device designs. The approach is very promising, but for the moment, the latter innovations do not cover cataract surgery and ophthalmology.

\section{Irrigation device design based on hydrodynamic analysis}

Based on mathematical modeling, surgical tool design can be carried out in a rational and validated manner. For example, it is known that sudden expansion of a channel is accompanied by significant hydraulic resistance i.e. decrease of the total flow pressure (Abramovich, 1969). The pressure drop in expanding stream of the irrigation fluid (in absence of a wall) may be evaluated as follows:

$$
\Delta p=\frac{\rho V_{+}^{2}}{2}\left(1-\frac{S_{+}}{S}\right)
$$

where $S$ is the surface area formed by the expanding stream (the sum of end surface areas of separate fluid jets). Consequently, the greater $S$ is, the smaller the hydrodynamic effect of the fluid on eye tissue is exerted.

The stream, which outflows from the tubule with normal (perpendicular to the axis) outlet cross section into a great volume cavity, has a shape nearing to a cone. If series of holes are made in the tubule wall and their number and arrangement are correctly chosen, an almost spherically expanding stream can be obtained, and consequently the maximal area $S$. In this case, it is possible to approach almost the minimal pressure value on the wall determined by the formula (16).

The idea has been embodied in a device for irrigation (Pletnev et al., 2005) intended for the bimanual phacoemulsification technique. The device contains the hollow tube 1 with the 
hooked end 2 as shown in Fig. 7. A part of cylindrical surface 2 has at least two rows of irrigation holes 3 . Each three of these holes are situated at the corners of equilateral triangles and form equilateral triangles with the neighbouring holes. The closed end surface 4 of the hooked end is furnished with at least one irrigation hole. The total area of all holes lies within the range $20 \%$ to $25 \%$ of the area of the cylindrical surface of the hooked end.

The device is designed so that the fluid outflows from irrigation holes in the form of fluid jets oriented normally to the cylindrical surface. In all holes, approximately the same flow rate and hydraulic drop are realized. The fluid jets interflowing form a closed frontal surface akin to spherical one, where hydrodynamic pressure is at the minimum.

An advanced device for irrigation which functions in a similar way is shown in Fig. 8 (Spirochkin et al., 2008). This device contains the hollow tube 1 with the closed end surface furnished with irrigation holes 2 . The end surface is designed in the form of an ellipsoid of revolution 3 extended along the tube axis. This form of the end surface is chosen to ensure nearly spherical front surface of the outflowing irrigation fluid and approximate equidistance to the crystalline lens inner surface inside which this end enters. The holes 2 are situated at equal spatial angles 4 measured relative to the ellipsoid center. The total area of the irrigation holes lies within the range $40 \%$ to $50 \%$ of the ellipsoid surface area.

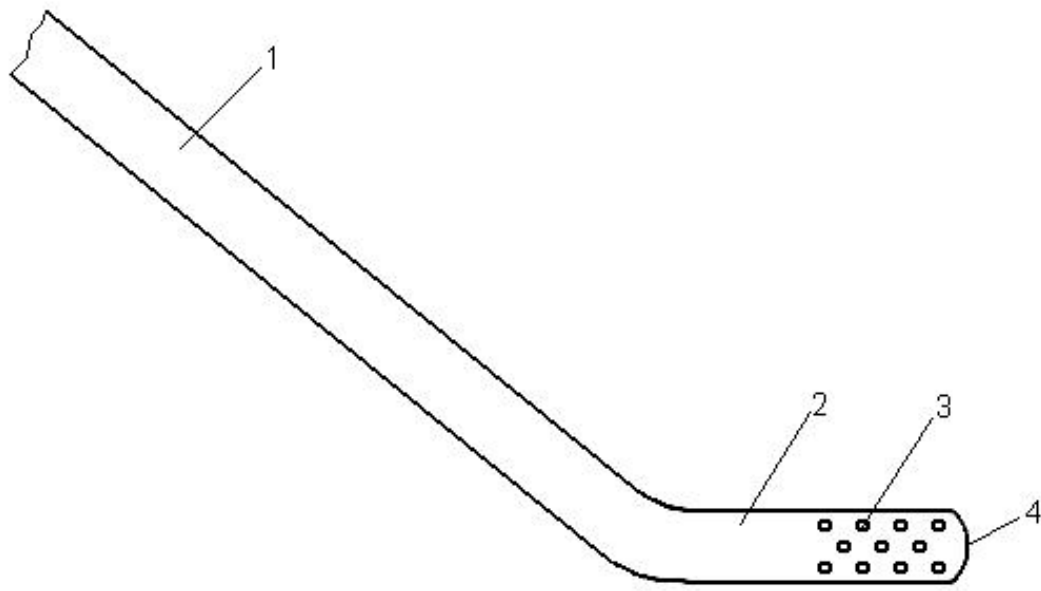

Fig. 7. Device for irrigation (Pletnev et al., 2005). See text for details. 
The irrigation fluid outflowing through the holes 2 forms a closed frontal ellipsoid surface and effects with nearly minimum hydrodynamic pressure on the internal surfaces of eye tissues. Hence use of these inventions theoretically reduces trauma to eye tissues.

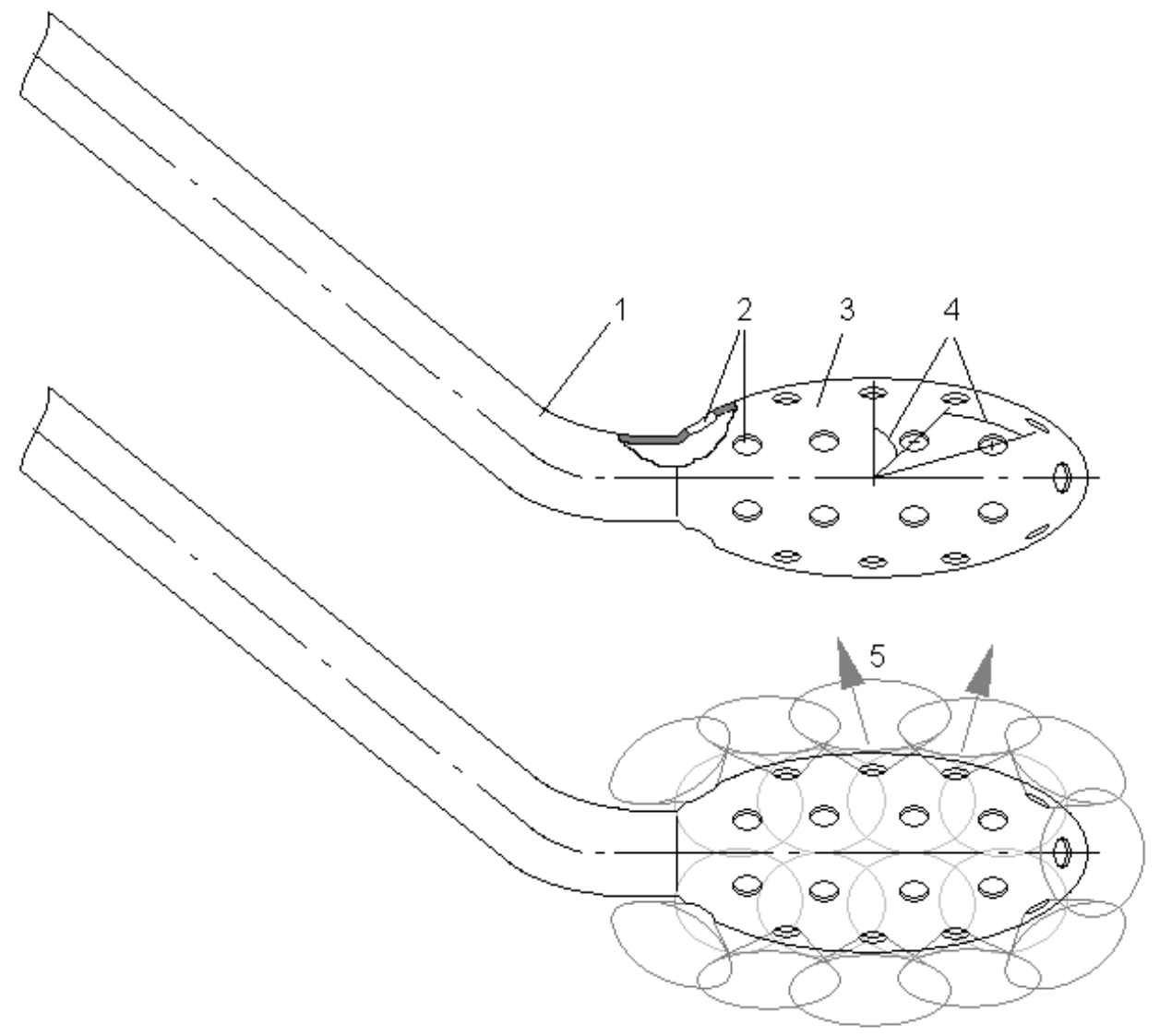

Fig. 8. Advanced device for irrigation (Spirochkin et al., 2008). See text for details.

\section{Conclusion}

The analytical approach, which has been presented, provides a rational basis for evaluation of new surgical methods in ophthalmology and for design of surgical tools. Simplified mathematical modeling of hydrodynamic processes during surgical operations is similar to the technique of structure dimensioning, or sizing, commonly used in engineering practice, e.g. in nuclear engineering (with which the author is especially familiar). Surgical procedures or structural parameters of surgical tools obtained at the stage of dimensioning can be checked and corrected at the next stage of engineering: coupled structure-fluid analysis using the finite element method. However techniques of finite element analysis for support of cataract surgery need to be developed. 


\section{Acknowledgment}

The problem has been introduced to the author by Dr. Zimina (Moscow). Her assistance during its formulation and solution is invaluable. The author is thankful to Mr. Pletnev, the head of experimental test laboratory in the Energia Corp. (Korolyov near Moscow), for enormous intellectual contribution to this work.

\section{References}

Abramovich, G.N (1969). Applied Gasdynamics (3rd Edition). - Moscow: Science (in Russian)

Amini, R. and Barocas, V.H. (2009). Anterior Chamber Angle Opening during Corneoscleral Indentation: The Mechanism of Whole Eye Globe Deformation and the Importance of the Limbus. Investigative Ophthalmology \& Visual Science. Vol. 50, No. 11, pp. 52885294

Aviation acoustics (1986). Ed. by A.G. Munin. In 2 parts. Part 2. - Moscow: Machinebuilding (in Russian)

Belezza, A.J.; Hart, R.T.; Burgoyne, C.F. (2000). The Optic Nerve Head as a Biomechanical Structure: Initial Finite Element Modeling. Investigative Ophthalmology \& Visual Science. Vol. 41, No. 10, pp. 2991-3000

Bryant, M.R.; Szerenyi, K., Schmotzer, H.; McDonnel, P.J. (1994). Corneal Tensile Strength in Fully Healed Radial Keratotomy Wounds. Investigative Ophthalmology \& Visual Science. Vol. 35, No. 7, pp. 3022-3031

Crouch, J.R.; Merriam, J.C.; Crouch, E.R. (2005). Finite Element Model of Cornea Deformation. Medical Image Computing and Computer-Assisted Intervention - MICCAI 2005. Lecture Notes in Computer Science. Vol. 3750/2005, pp. 591-598, Available from: http://www.springerlink.com/content/3vc2ua4tjqdf2vw9

Elastohydrodynamics of the Anterior Eye (2002). Supercomputing Institute Research Bulletin. Summer 2002. University of Minnesota. Vol. 18, No. 2, Available from: http://static.msi.umn.edu/research_bulletin/Vol.18-No.2

Girard, M.J.A.; Suh, J.-K.F; Bottlang, M.; Burgoyne, C.F.; Downs, J.C. (2009). Scleral Biomechanics in the Aging Monkey Eye. Investigative Ophthalmology \& Visual Science. Vol. 50, No. 11, pp. 5226-5237

Landau, L.D. and Livshits, E.M. (1988). Hydrodynamics. - Moscow: Science (in Russian)

Malyugin, B.E. (2002). Medical and Technological System for Surgical Rehabilitation of Patients with Cataract on the Base of Ultrasonic Phacoemulsification and Intraoculare Lens Implantation.Dissertation. - Moscow: Fedorov's Center of Eye Microsurgery (in Russian)

Medical Device Invention and Evaluation Through Simulation, Visualization, and Interactive Design (2010). Supercomputing Institute Research Bulletin. Fall 2010. University of Minnesota. Vol. 26, No. 3, Available from:

http:/ / static.msi.umn.edu/research_bulletin/2010-26-3.pdf

Pinsky, P. and Datye, D. (1991). A Microstructurally Based Finite Element Model of the Incised Human Cornea. Journal of Biomechanics, Vol. 24, pp. 907-992

Pletnev, I.V.; Spirochkin, Yu.K.; Zimina, T.Yu., Trubilin, V.N. (2005). Device for Irrigation. Patent of Russian Federation for useful model, No. 51869 (in Russian) 
Poloz, M.V.; Iomdina, E.N., Spirochkin, Yu.K. (2008). Numerical Modeling for Optical and Mechanical Proccesses of Accomodation, Available from: http://www.helmholtzeyeinstitute.ru/photo/406.doc

Sigal, I.A.; Flanagan, J.G.; Tertinegg, I.; Ethier, C.R. (2004). Finite Element Modeling of Optic Nerve Head Biomechanics. Investigative Ophthalmology \& Visual Science. Vol. 45, No. 12, pp. 4378-4387

Spirochkin, Yu.K.; Takhchidi, Kh.P., Karavaev, A.A. (2008). Device for Irrigation. Patent of Russian Federation for invention, No. 2331395 (in Russian)

Trubilin, V.N; Zimina T.Y., Spirochkin, Yu.K. (2005). Comparative Analysis of Hydrodynamic Streams during the Coaxial and the Bimanual Phacoemulsification. Refractive Surgery and Ophthalmology, Vol. 5 (No.1), pp. 19-25 (in Russian)

Uchio, E.; Ohno, S.; Kudoh, J.; Aoki, K.; Kisielewicz, L. (1999). Simulation Model of an Eyeball Based on Finite Element Analysis on a Supercomputer. British Journal of Ophthalmology, Vol. 83, pp. 1106-1111 